\title{
Análisis socioeconómico y fitosanitario de fincas de producción de chiltoma en Tisma, Masaya, 2019
}

\author{
Socioeconomic and phytosanitary analysis of green pepper \\ production farms (Capsicum annuum L.) in Tisma, Masaya, 2019
}

\author{
Edgardo Jiménez Martínez ${ }^{1}$ \\ Carlos Alberto Cardoza Gonzalez ${ }^{2}$ \\ José María Roque García ${ }^{3}$
}

\section{Resumen}

En Nicaragua la producción de chiltoma (Capsicum annuum L.) está en manos de pequeños y medianos agricultores principalmente en el norte del país, para el ciclo 2017-2018 el Banco Central dio a conocer que en Nicaragua se establecieron 562 ha-1, con una producción de 18,182 toneladas y rendimientos de 32,3 $t$ ha-1. En Tisma no se ha realizado anteriormente ningún estudio acerca de las condiciones socioeconómicas y fitosanitarias en las que se encuentran los sistemas de producción de chiltoma. Esta investigación tuvo por objetivo caracterizar sistemas de esta hortaliza desde el punto de vista socioeconómico, fitosanitario, mediante la aplicación de una encuesta a productores. En base a la información recopilada, se ha interpretado el registro de 20 sistemas de producción de este pimiento. Los resultados mostraron que la mayoría de productores de chiltoma corresponden al sexo masculino, con edades entre 30 y 74 años, la mayoría están en manos de sus propios dueños y otra parte son alquiladas. Los principales insectos y ácaros plagas presentes, son los ácaros, y la mosca blanca (Bemisia tabaci), la enfermedad que más se presenta es la virosis, seguida de la marchitez por Phytophthora, las malezas más reportadas son el coyolillo (Cyperus rotundus L.) y bledo (Amaranthus spinosus L.). Los insectos son manejados en su mayoría haciendo uso de insecticidas químicos sintéticos. El manejo de las enfermedades de suelo, follaje y fruto, en su totalidad se realiza mediante el uso de químicos sintéticos. Mayormente las malezas son manejadas mediante prácticas culturales como uso de machete y azadón.

Palabras claves: Productores; Hortalizas; Manejo de plagas; Nicaragua.

\section{Abstract}

In Nicaragua the production of sweet pepper (Capsicum annuum L.) is in the hands of small and medium farmers mainly in the north of the country, for the 2017-2018 cycle the Central Bank reported that 562 ha-1 were established in Nicaragua, with a production of 18,182 tons and yields of $32.3 \mathrm{t}$ ha-1. In Tisma, no study has been previously conducted on the socioeconomic and phytosanitary conditions in which the sweet pepper production systems are found. The objective of this research was to characterize systems of this vegetable from the socioeconomic and phytosanitary point of view, through the application of a survey to producers. Based on the information collected, the registry of 20 production systems of this

\footnotetext{
${ }^{1} \mathrm{PhD}$ en Entomología, docente investigador, director de investigación, extensión y posgrado, Universidad Nacional Agraria, Nicaragua, Correo: edgardo.jimenez@ci.una.edu.ni ORCID: https://orcid.org/0000-0003-1086-7380

2 Ingeniero Agrónomo, Universidad Nacional Agraria, Nicaragua, Correo: cardozagonzalez6@yahoo.es

3 Ingeniero Agrónomo, Universidad Nacional Agraria, Nicaragua, Correo: jose.roque18@yahoo.es
} 
bell pepper was interpreted. The results showed that the majority of chiltoma producers are male, aged between 30 and 74 years, most are in the hands of their own owners and some are rented. The main insect and mite pests present are mites and whiteflies (Bemisia tabaci), the most common disease is virosis, followed by Phytophthora wilt, and the most reported weeds are coyolillo (Cyperus rotundus L.) and bledo (Amaranthus spinosus L.). Insects are mostly managed using synthetic chemical insecticides. Soil, foliage and fruit diseases are managed entirely with synthetic chemicals. Weeds are mostly managed by cultural practices such as machete and hoeing.

Keywords: Producers; Vegetables, Pest Control; Nicaragua.

\section{Introducción}

La chiltoma (Capsicum annuum L.) Pertenece a la Familia solanácea, es una hortaliza bien importante por su valor nutritivo, es rica en vitaminas A, B1, B2 y C (Laguna et al., 2006). Es una planta originaria de regiones tropicales específicamente de Bolivia y Perú. De estos países se propagó para la mayor parte de los países del continente americano, se adaptó en las diferentes regiones como el sur de Brasil, México y luego en el resto del mundo (CATIE, 1993).

La producción mundial de chiltoma o pimiento en 2016 fue de 34.497 millones de $\mathrm{kg}$. China es el mayor productor mundial con 17.435 millones de kg equivalente al 50.34\% del total. Seguido de México con 2.737 millones de kg, Turquía con 2.457, Indonesia con 1.961 y España con 1.082 millones de kg (FAO, 2014).

En Nicaragua la producción de chiltoma está en manos de pequeños y medianos agricultores principalmente de la zona norte del país, la forma de consumo fresco, es ampliamente utilizada para condimentar toda clase de alimentos. (Laguna et al., 2004). Para el ciclo 2017-2018 el Banco Central dio a conocer que en Nicaragua se establecieron 562 ha-1, con una producción de 18182 toneladas (6.8\% de crecimiento) y rendimientos de 32.3 t ha-1. Se registró un consumo aparente de 17727 toneladas (BCN, 2017).

Muchas áreas del municipio de Tisma se encuentran altamente contaminadas con plaguicidas químicos, y esto se debe a que el $95 \%$ de los agricultores de la zona desde hace muchos años han utilizado excesivamente los productos químicos sintéticos para combatir los organismos plagas. Esto ha traído efectos negativos en la fauna benéfica, salud humana y el medio ambiente.

Entre 2010-2013, se llegó a conocer que en la zona de Tisma, el cultivo de la chiltoma fue atacado fuertemente por ácaros, el cual ocasionó daños muy severos en los rendimientos, con pérdidas hasta de un 50 \% (Barberena y Lacayo, 2011).

En el municipio de Tisma no se ha realizado ningún estudio de las condiciones socioeconómicas y fitosanitarias en las que se encuentran los sistemas de producción de esta hortaliza y no se tiene conocimiento específico de la situación actual, esto no ha permitido que instituciones del estado, ONG's, empresas privadas puedan impulsar proyectos con fines a desarrollar y mejorar la situación económica y productivas.

El presente estudio caracteriza desde el punto de vista socioeconómico, agronómico y fitosanitario, veinte unidades de producción del cultivo de chiltoma en el municipio de Tisma, Masaya. Los resultados de este estudio le permitirán a instituciones del Estado, empresa privada, ONG's, u otras, formular planes estratégicos para mejorar las condiciones socioeconómicas y productivas de productores de esta zona. 


\section{Materiales y métodos}

\section{Descripción del área de estudio}

El estudio se llevó a cabo en el municipio de Tisma, departamento de Masaya, que se ubica a $36 \mathrm{~km}$ de Managua, se encuentra en las coordenadas $12^{\circ} \mathrm{o} 4^{\prime}$ oo" latitud norte y $86^{\circ} \mathrm{o} 1^{\prime}$ oo" longitud oeste, limita al norte con el municipio de Tipitapa, al suroeste con el municipio de Masaya y al este con el municipio de Granada. Presenta una altitud de $50 \mathrm{msnm}$, con un clima tropical de sabana y una temperatura promedio de $27.5^{\circ} \mathrm{C}$. Cuenta con 11 comunidades en las cuales se cultivan hortalizas y granos básicos (Castillo, 2017). La etapa de campo del estudio se realizó a partir del 3 al 15 de abril, la etapa de gabinete (redacción) se inició el 20 de abril al 10 de agosto de 2019.

\section{Diseño metodológico}

El estudio desarrollado fue de naturaleza no experimental del tipo transversal, exploratorio. Consistió en visitas a las áreas de producción y con la ayuda de productores claves, mediante el método descriptivo se realizó una caracterización socioeconómica y fitosanitaria de los sistemas de producción de chiltoma en Tisma, la aplicación de este método es necesario para medir las distintas variables aplicadas en el estudio realizado.

\section{Metodología para la obtención de la información}

La metodología empleada consistió en el uso de un modelo de desarrollo participativo con productores de la zona, el cual se implementó haciendo uso de la técnica de entrevista personal, para el análisis de la información se hizo uso de la estadística descriptiva, la cual es una parte de la estadística que se dedica al análisis y representación de datos. Este estudio se realizó en tres fases las que se describen a continuación:

- Primera etapa (Fase 1): Se definió la muestra a utilizar y los sistemas de producción en que se realizó el estudio, se seleccionó un máximo de 20 unidades productivas, dedicadas a cultivar chiltoma en el municipio de Tisma, así mismo se realizaron visitas a instituciones como: INTA, IPSA, Alcaldía. Todo esto en busca de información referente a la cantidad de productores en Tisma y cuántos de ellos cultivan chiltoma, de igual manera se buscó información referente al municipio, sitios web y cualquier otra fuente de información que nos conllevara a caracterizar los sistemas sociales y productivos de la zona de estudio. Esta información encontrada también nos ayudó a la elaboración de las encuestas y a la estructura del informe.

- Segunda etapa (Fase 2): Se procedió a recopilar la información socioeconómica y fitosanitaria de los productores de chiltoma de Tisma, la información recopilada consistió en variables del tipo socioeconómico, agronómico y fitosanitario, a través de una encuesta.

- Tercera etapa (Fase 3): Se realizó el ordenamiento y procesamiento de la información procedente de las encuestas y de la visita a cada unidad de producción. También se involucró la actividad de escritura del documento final.

\section{Instrumento utilizado para la recolección de la información}

Partiendo de los objetivos establecidos se estructuró como instrumento de colecta de datos en campo una encuesta, la cual se aplicó a los productores en cada una de las fincas visitadas. Los datos resultantes 
sirvieron de referencia para determinar el estado en que se encuentran actualmente los sistemas productivos de chiltoma en el municipio de Tisma. Así mismo se solicitó una visita a las áreas donde se encuentra el cultivo.

\section{Variables evaluadas}

Cuadro 1. Descripción de las variables utilizadas en la caracterización de sistemas de producción de chiltoma en Tisma, Masaya, 2019

\begin{tabular}{|c|c|}
\hline Variables & Metodología empleada \\
\hline Edad: & Tomada en años a cada uno de los productores. \\
\hline Sexo: & Preguntando el sexo al dueño de la finca. \\
\hline Tamaño de la finca: mz & Preguntando directamente al productor por tamaño. \\
\hline Tenencia de la tierra: & Preguntándole al productor el estado legal la finca \\
\hline Estado de la vivienda & $\begin{array}{l}\text { Mediante la observación directa de la vivienda del productor } \\
\text { y haciendo preguntas al mismo. }\end{array}$ \\
\hline $\begin{array}{l}\text { Principales insectos y ácaros } \\
\text { plagas que afectan la chil- } \\
\text { toma. }\end{array}$ & $\begin{array}{l}\text { Consultando directamente al productor si conoce las plagas } \\
\text { que afectan el cultivo de chiltoma. }\end{array}$ \\
\hline Manejo de plagas del suelo & $\begin{array}{l}\text { Preguntando directamente al productor como maneja las } \\
\text { plagas en la chiltoma. }\end{array}$ \\
\hline Manejo de plagas del follaje & $\begin{array}{l}\text { Preguntando directamente al productor como maneja las } \\
\text { plagas en la chiltoma. }\end{array}$ \\
\hline Manejo de plagas del fruto & $\begin{array}{l}\text { Preguntando directamente al productor como maneja las } \\
\text { plagas en la chiltoma. }\end{array}$ \\
\hline $\begin{array}{l}\text { Principales enfermedades } \\
\text { que afectan la chiltoma }\end{array}$ & $\begin{array}{l}\text { Consultando directamente al productor si conoce las enfer- } \\
\text { medades que afectan el cultivo de chiltoma. }\end{array}$ \\
\hline $\begin{array}{l}\text { Manejo de enfermedades } \\
\text { del suelo, follaje y fruto }\end{array}$ & $\begin{array}{l}\text { Preguntando directamente al productor como maneja las } \\
\text { enfermedades en la chiltoma. }\end{array}$ \\
\hline $\begin{array}{l}\text { Principales malezas que } \\
\text { afectan la chiltoma }\end{array}$ & $\begin{array}{l}\text { Consultando directamente al productor si conoce las male- } \\
\text { zas que afectan el cultivo de chiltoma }\end{array}$ \\
\hline Manejo de malezas & $\begin{array}{l}\text { Preguntando directamente al productor como maneja las } \\
\text { malezas en la chiltoma. }\end{array}$ \\
\hline
\end{tabular}

\section{Análisis de los datos}

Se realizó una base de datos conformadas por variables categóricas y cuantitativas, luego se procesó en hojas electrónicas (Excel), también fueron utilizadas para cada variable técnicas estadísticas básicas tales como medidas de posición y tablas de contingencias.

\section{Resultados y discusión}

\section{Edad de los productores}

Nicaragua es un país de gente mayoritariamente joven. La población menor de 19 años, que incluye a niñas, niños y adolescentes representan el $38.7 \%$, de la población total del país que equivale a 6.2 millones de habitantes (INIDE, 2014).

En cada una de las unidades de producción visitadas se encuestó al productor, se encontró que en un $25 \%$, el rango de edad de los productores anda entre los 30-34 años, seguido de las edades entre 60-64 con un 20 $\%$, y un $15 \%$ representa las edades entre 70 y 74 años, esto indica que la mayoría de productores de chiltoma 
en Tisma son mayores de los 30 años y menores a los 74 años (Figura 1). Castillo (2017) encontró que, la edad de los productores de solanáceas en Tisma, generalmente oscila en un rango entre los 30 y 56 años.

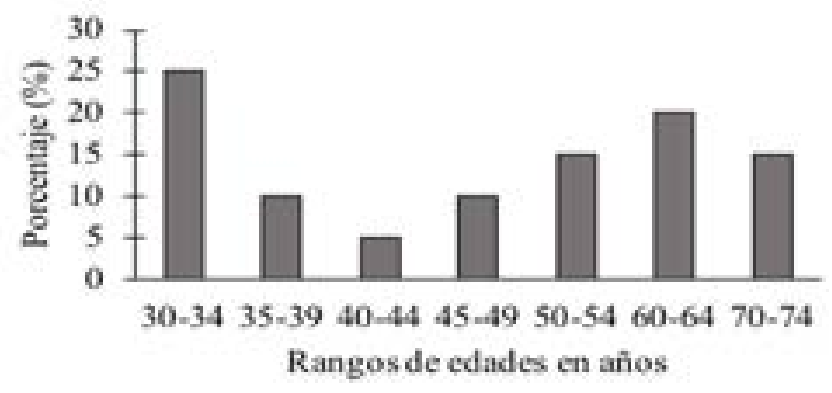

Figura 1. Rango de edades de los productores de chiltoma

\section{Tenencia de la propiedad}

La Figura 3 muestra la distribución de las unidades productivas de chiltoma de acuerdo a la tenencia de la propiedad, donde el $35 \%$ de las unidades de producción son manejadas por sus propietarios, otro $35 \%$ son tierras alquiladas, un $15 \%$ pertenecen a cooperativas y el restante $15 \%$ son prestadas. Entre los propietarios se encuentran productores con muchos años produciendo chiltoma.

Según INIDE (2011) indica que el tema del derecho a la propiedad y tenencia de la tierra, encierra parte de un estado democrático, el cual debe estar gobernado por leyes acordadas entre todos, en el que se reconocen y respetan la libertad individual a la propiedad y la tenencia de la tierra.

Nicaragua cuenta con un total de 394068 explotaciones agropecuarias, de las cuales el $89 \%$ están en manos de sus propios dueños, seguidamente se ubica la tenencia de alquiler con un 5.3 \% INIDE (2011).

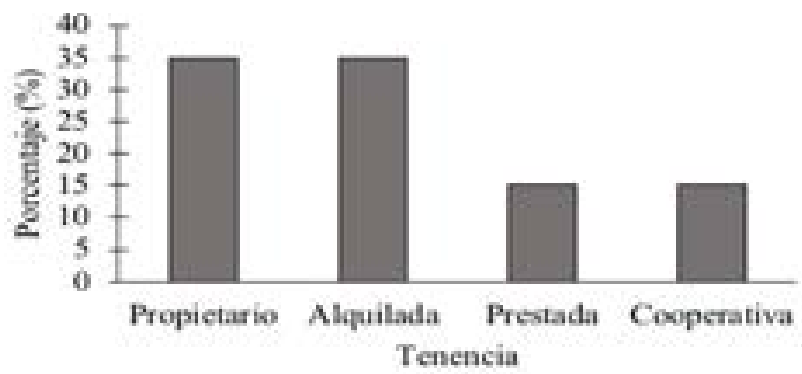

Figura 3. Tenencia de la propiedad de las unidades de producción

\section{Tamaño de las unidades de producción}

En la Figura 4, se muestra la distribución de las unidades productivas de chiltoma de acuerdo a su tamaño en manzanas en el municipio de Tisma, donde el $60 \%$ de los productores cultivan chiltoma en superficie inferiores a $1 \mathrm{mz}$ y el restante $40 \%$ lo hace en áreas entre $1.1-2 \mathrm{mz}$, esto demuestra que los sistemas de 
producción de chiltoma están siendo manejadas a pequeña escala (pequeños productores), este resultado coincide con los datos reportados en el censo nacional agropecuario INIDE (2011), en donde se menciona que el $80 \%$ de los productores del país son tipificados como pequeños.

De acuerdo a Calero (2014), la escasez de tierra debido a la distribución desigual y al crecimiento de poblaciones rurales, está obligando a que los productores subdividan sus parcelas entre los miembros de la familia, lo que provoca una marcada reducción en la relación tierra/persona.

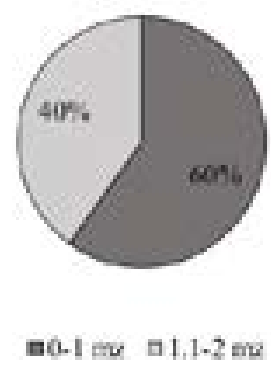

Figura 4. Distribución las unidades de producción de chiltoma según en tamaño del área en manzanas

\section{Principales insectos y ácaros plagas en chiltoma}

En la Figura 5, se muestran resultados de los insectos y ácaros plagas que más afectan las plantaciones de chiltoma en Tisma. Encontrándose a los ácaros (Polyphagotarsonemus latus) en el $100 \%$ de las unidades productivas, seguido de la mosca blanca (Bemisia tabaci) con presencia en el $95 \%$ de los sistemas de producción y el $80 \%$ de los productores reportaron minadores (Phyllocnistis citrella), los que menos fueron detectados son Spodoptera spp y gallina ciega (Phyllophaga spp) con un $55 \%$. Esto se relaciona a lo descrito por Barberena y Lacayo (2011) en un estudio en chiltoma realizado en Tisma, se comparó la fluctuación poblacional de ácaros, mosca blanca y áfidos, desde los $7 \mathrm{ddt}$ hasta los $92 \mathrm{ddt}$, se observaron poblaciones de ácaros desde los $7 \mathrm{ddt}$, y su mayor población fue a los $63 \mathrm{ddt}$, las moscas blancas fueron visualizadas desde las primeras fechas de muestreo y la mayor presencia de las mismas fue a los 42, 70 y $92 \mathrm{ddt}$.

Según Hernández (2016) en un estudio realizado en la finca El Plantel Masaya en el cultivo de chiltoma, mostró que las poblaciones de mosca blanca aparecieron desde los primeros $5 \mathrm{ddt}$, pero a partir de los 51 ddt las poblaciones de mosca blanca aumentaron de forma progresiva a través del tiempo, mantuvieron su nivel poblacional por encima del nivel crítico en ambos tratamientos, surcos sencillos y dobles con más de 1 ninfa y 1 adulto de mosca blanca por planta.

De acuerdo a Jiménez Martínez (2007),la mosca blanca es la plaga más importante en la transmisión de virus en las solanáceas, succionan la savia de las plantas, causando problemas como la producción de hongos que crecen sobre la melaza, que excretan y bloquean la luz del sol necesaria para las plantas, reduciendo la producción y llegando a dañar o matarlas, pero el principal daño ocurre cuando llevan virus a las plantas sanas después de haberse alimentado en una planta enferma. 


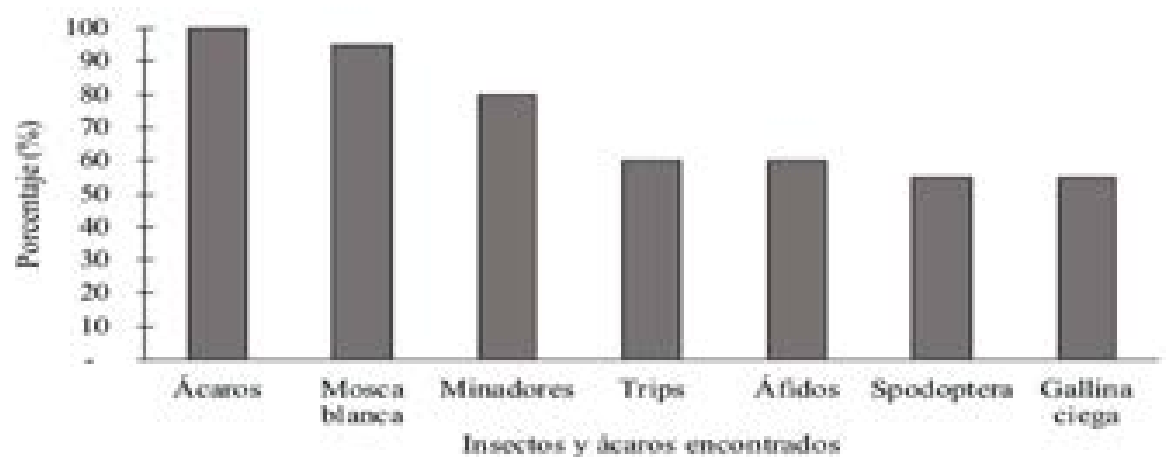

Figura 5. Insectos y ácaros plagas encontrados en el cultivo de chiltoma

\section{Manejo de insectos plagas del follaje en chiltoma}

Se muestra el tipo de manejo de plagas del follaje que realizan los productores de chiltoma, encontrándose que el $100 \%$ de los productores prefieren emplear el método químico para el control de estas, en vez del uso del control botánico, biológico o cultural. Dentro del control quimico un $30 \%(n=6)$ de los productores menciona que tiene inclinación a aplicar Engeo ${ }^{\circledR} 24.7$ SC (tiametoxam+lambda cyalotrina), seguido del 20 $\%(\mathrm{n}=4)$ que usan Imidacloprid ${ }^{\circledR} 35$ SC (1-(6-chloro-3-pyridylmethyl)-N-nitroimidazolidin-2-ylideneamine) y el restante $50 \%$ utilizan otros tipos de insecticidas.

En un estudio realizado por Martínez y Jirón (2011) en Tisma departamento Masaya, determinaron que el uso del Oberón resultó más efectivo para el manejo del ácaro blanco seguido por los tratamientos Vertimec y Chile + jabón en el cultivo de chiltoma, ya que las plantas que fueron tratadas con estos productos presentaron las menores poblaciones, menor incidencia y severidad de daño ácaro blanco.

\section{Manejo de plagas insectiles del fruto en chiltoma}

El $100 \%$ de los productores de chiltoma prefieren utilizar el método químico para el control de plagas insectiles de los frutos, donde el $75 \%$ emplea Coragen ${ }^{\circledR} 20$ SC (3-bromo-4冈-cloro-1-(3-cloro-2-piridil)-2区metil-6冈- (metilcarbamoil) pirazol-5-carboxanilida) y el restante $25 \%$ utilizan Abamectina ${ }^{\circledR}\left(\mathrm{C}_{4} 8 \mathrm{H}_{72} \mathrm{O}_{14}\right.$ (avermectinB1a)). De acuerdo a Castillo (2017) el 35 \% de los productores de solanáceas utilizan Coragen ${ }^{\circledR}$ 20 SC para controlar plagas del fruto.

\section{Principales enfermedades encontradas}

En la Figura 7, se muestran las principales enfermedades que más afectan las plantaciones de chiltoma en Tisma de acuerdo a lo reportado por los productores, encontrándose que el $100 \%(\mathrm{n}=20)$ de las unidades de producción son afectados por virosis, un $55 \%(n=11)$ de productores reportaron afectaciones de marchitez causado por (Phytophthora spp) y un $15 \%(\mathrm{n}=3)$ de las áreas en estudio reportan problemas por mancha foliar causada por Alternaría. De acuerdo a Guigón y González (2001) indican que es reconocida la susceptibilidad de este cultivo a numerosas enfermedades que afectan la calidad y los rendimientos. En los últimos años, esta hortaliza ha recibido gran atención en el mundo entero debido al ataque de plagas y enfermedades que afectan este rubro. 


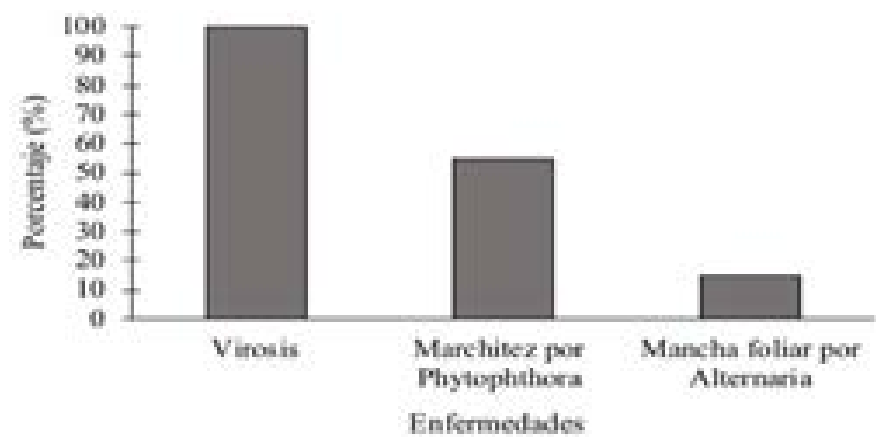

Figura 7. Enfermedades que afectan el cultivo de chiltoma

\section{Principales malezas que afectan a la chiltoma}

En el Cuadro 3 se muestran las principales malezas encontradas en el cultivo de chiltoma, donde los productores de Tisma reportan al coyolillo (Cyperus rotundus L.) en un $80 \%(n=16)$ de las unidades productivas como la maleza más común que afecta al cultivo de la chiltoma, seguido del bledo (Amaranthus spinosus L.) que fue reportada por el $70 \%(n=14)$ de productores y la pata de gallina (Digitaria sanguinalis (L) scop.) tuvo presencia en el $65 \%(\mathrm{n}=13)$ de las áreas en estudio, las que menos fueron encontradas son zacate dulce (Ixophorus unicetus (J. Presl)) y zacate peludo (Rottboellia cochinchinensis (Lour.) Clayton). Altas poblaciones de malezas pueden disminuir el rendimiento de los cultivos entre un 20 a $60 \%$, aunque algunos autores reportan hasta un $85 \%$ de mermas en los rendimientos dependiendo del tipo de cultivo, época de siembra y densidad de población (INTAGRI a, 2017).

Las arvenses presentes en el cultivo de chiltoma representan un riesgo importante, dado que compiten con el cultivo de interés por luz, agua, espacio y nutriente, ofreciendo refugio a insectos plagas que afectan al mismo. El coyolillo destaca por ser una maleza invasora, es decir, se encuentra en todos los campos agrícolas del mundo y es considerada una de las arvenses de mayor importancia económica en los cultivos a nivel mundial. Es una arvense con alta capacidad de propagación al reproducirse por semilla, rizomas y tubérculos. Tiene un rápido crecimiento vegetativo y es capaz de producir sustancias alelopáticas (INTAGRI a, 2017).

Cuadro 3. Malezas más comunes reportadas en fincas de chiltoma

\begin{tabular}{|l|l|l|}
\hline \multirow{2}{*}{ Tipos de maleza } & \multicolumn{2}{c|}{ Fincas que reportan tipos de malezas en chiltoma } \\
\cline { 2 - 3 } & \multicolumn{1}{|c|}{ Frecuencia } & \multicolumn{1}{c|}{$\%$} \\
\hline Coyolillo & 16 & 80 \\
\hline Bledo & 14 & 70 \\
\hline Pata de gallina & 13 & 65 \\
\hline Flor amarilla & 2 & 10 \\
\hline Zacate dulce & 1 & 5 \\
\hline Zacate peludo & 1 & 5 \\
\hline
\end{tabular}




\section{Manejo de malezas en el cultivo de chiltoma}

En la Figura 8, se muestra el tipo de manejo de malezas más empleado en las fincas de chiltoma, se encontró que el $65 \%$ de los productores de Tisma realizan mayoritariamente prácticas culturales, entre ellas el uso del machete, azadón, quema, entre otras y el restante $35 \%$ prefiere utilizar el método químico, de este método el $86 \%(n=6)$ usa Paraquat ${ }^{\circledR}$ (Dicloruro de 1,1'-dimetil-4,4'- bipiridilo), y el $14 \%(n=1)$ emplea Yerbalade $^{\circledR} 15$ EC (fluazifop-p-butil).

Marín y Berrouet (2016) mencionan que, el paraquat ${ }^{\circledR}$ (Dicloruro de 1,1'-dimetil-4,4'- bipiridilo) es un herbicida de rápida acción y actúa en las hojas por contacto directo. Está clasificado como una sustancia de gran toxicidad tras su ingestión. Por su alta toxicidad el uso de este producto antes mencionado está prohibido en los Estados Unidos y en muchos países europeos.

De acuerdo a INTAGRI b (2017), la forma más adecuada de controlar las malezas consiste en establecer programas de manejo integrado basados en las siguientes herramientas: identificación correcta de las malezas, conocimiento del historial del sistema de producción, monitoreo continuo de los predios y áreas aledañas, estrategias de control basadas en las poblaciones y diversidad de malezas mediante una combinación de técnicas, uso adecuado de herbicidas, monitoreo y evaluación de la eficiencia de las decisiones de manejo.

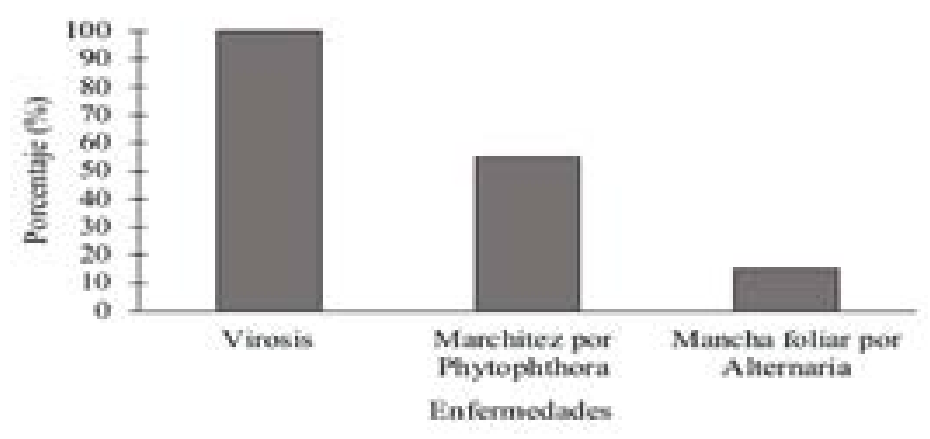

Figura 8. Tipo de manejo de malezas más empleado en el cultivo

\section{Conclusiones}

La mayor parte de los productores de chiltoma de Tisma pertenecen al sexo masculino, la edad de los mismos oscila entre los 30 a 74 años. La mayoría de las tierras son propias y otra parte son alquiladas, el tamaño de las unidades de producción es inferior a 2 manzanas. El estado de la vivienda está entre buenas y muy buenas.

Los ácaros son la principal plaga de la chiltoma, seguido de la mosca blanca, las enfermedades que más afecta los sistemas de producción son las virosis, seguido de la marchitez por Phytophthora, Las malezas predominantes son el coyolillo y el bledo.

Los productos químicos sintéticos son los más usados para el manejo de las plagas insectiles, Vydate ${ }^{\circledR}$ para el manejo de plagas del suelo, Engeo ${ }^{\circledR}$ para el manejo de plagas del follaje, y plagas del fruto emplean Coragen $^{\circledR}$, las enfermedades son manejadas con químicos sintéticos. El control de malezas lo realizan empleando prácticas culturales como el uso de machete, azadón. 


\section{Lista de referencias}

Barberena Moncada, J. A., y Lacayo Narváez, Y. A. (2011). Evaluación de alternativas botánicas y químicas para el manejo del acaro blanco (Poliphagotarsonemus latus, Bank)(Acarina: Tarsonemidae) e insectos plagas en el cultivo de chiltoma (Capsicum annuum L.) Tisma, Masaya. (Tesis de grado). Recuperada de http://repositorio.una.edu.ni/2133/1/tnh1ob234.pdf

Calero Chavarría E. R. (2014). Características básicas de Unidades Familiares Productivas en las comunidades de Nueva Esperanza y Buena Vista, en la Reserva Natural Tepec-Xomolth La Patasta. Las Sabanas. Recuperado de http://repositorio.una.edu.ni/3161/1/tnpo1c149.pdf

Castillo Martínez, L. I. (2017). Caracterización de los sistemas de producción del cultivo de tomate (solanum lycopersicum m.) en el municipio de Tisma, Masaya, Nicaragua, 2016. (Tesis de maestría). Recuperada de http://repositorio.una.edu.ni/360o/1/tnfo1c352c.pdf

Centro agronómico tropical de investigación y enseñanza (1993). Guía para el manejo integrado de plagas del cultivo del chile dulce. Turrialba, Costa Rica: CATIE.

Guigón, C., y Gonzales, P. (septiembre, 2001). Estudio Regional de las Enfermedades del chile (Capsicum annuum, L). Revista Mexicana de Fitopatología 19(1), pp49-56. Recuperado de https://www.researchgate.net/publication/237037399_Estudio_Regional_de_las_Enfermedades_del_Chile_Capsicum_ annuum_L_y_su_Comportamiento_Temporal_en_el_Sur_de_Chihuahua_Mexico

Hernández Umanzor, E. J. (2016). Ocurrencia de mosca blanca (Bemisia tabaci Genn.) (Hemíptera: Aleyrodidae) e incidencia de virosis en chiltoma (Capsicum annuum L.) con dos densidades de siembra, en condición semiprotegida. (Tesis de grado).

Instituto Nacional de Información de Desarrollo (2014). Encuesta nicaragüense demográfica y salud INIDE-ENDESA. Recuperado de https://nicaragua.unfpa.org/sites/default/files/pub-pdf/ENDESA2011-12-completa.pdf

INTAGRI (2017a). El Manejo Integrado del Coquillo en Cultivos Intensivos. Ciudad de México, México: INTAGRI

INTAGRI. (2017b). El Manejo Integrado del Coquillo en Cultivos Intensivos. Recuperado de https://www. intagri.com/articulos/fitosanidad/el-manejo-integrado-de-coquillo-en-cultivos-intensivos

Jiménez Martínez, E. S. (2007). Guía de manejo integrado de mosca blanca y virus en Nicaragua. Recuperado de http://repositorio.una.edu.ni/2445/1/nh10j61g.pdf

Jiménez Martínez, E. S. (2009). Métodos de control de plagas. Recuperado de http://repositorio.una.edu. ni/2457/1/nh10j61c.pdf

Laguna, T., Gutiérrez, C., y Sarría, M. (2006). Guía tecnológica de chiltoma: Cultivo de la chiltoma. Recuperado de http://www.inta.gob.ni/biblioteca/images/pdf/guias/Guia\%20Chiltoma\%202014. pdf

Laguna, T., Pavón, J., y Nicaragua, K. (2004). Manejo integrado de plagas: Cultivo de la chiltoma. Recuperado de http://cenida.una.edu.ni/relectronicos/RENH1oL181.pdf 